DOI 10.4171/JEMS/260

Sönke Rollenske

\title{
The Kuranishi space of complex parallelisable nilmanifolds
}

Received July 10, 2008 and in revised form December 1, 2008

\begin{abstract}
We show that the deformation space of complex parallelisable nilmanifolds can be described by polynomial equations but is almost never smooth. This is remarkable since these manifolds have trivial canonical bundle and are holomorphic symplectic in even dimension. We describe the Kuranishi space in detail in several examples and also analyse when small deformations remain complex parallelisable.
\end{abstract}

\section{Introduction}

Left-invariant geometric structures on nilmanifolds, i.e., compact quotients of (real) nilpotent Lie groups, have proved to be both very rich and accessible for an in depth study. Thus many examples and counter-examples in (complex) differential geometry are of this type.

In this paper we want to study deformations of nilmanifolds which are complex parallelisable, that is, have trivial holomorphic tangent bundle. By a result of Wang [Wan54], all compact complex parallelisable manifolds arise as quotients of complex Lie groups.

The study of deformations of complex structures on compact complex manifolds has been an important topic since the work of Kodaira and Spencer in [KS58]. In particular they showed that first order deformations correspond to elements in $H^{1}\left(X, \Theta_{X}\right)$ where $\Theta_{X}$ is the sheaf of holomorphic tangent vectors.

In this paper we will look at deformations from the following point of view: consider $X$ as a differentiable manifold together with an integrable almost complex structure $(M, J)$, i.e., $J: T M \rightarrow T M, J^{2}=-\mathrm{Id}_{T M}$ and the Nijenhuis integrability condition holds (see (1) below). A deformation of $X$ can be given as a family of such complex structures $\left(J_{t}\right)_{t \in \mathcal{B}}$ parametrised by a complex analytic space $\mathcal{B}$ such that $J=J_{0}$ for some point $0 \in \mathcal{B}$.

A key result is the theorem of Kuranishi [Kur62] which guarantees the existence of a locally complete space of deformations $\operatorname{Kur}(X)$ which is versal at 0 . In other words, the Kuranishi family parametrises all sufficiently small deformations effectively, though not uniquely, which is roughly due to the existence of automorphisms. The construction of

S. Rollenske: Institut für Mathematik, Johannes-Gutenberg-Universität Mainz, Staudingerweg 9, 55099 Mainz, Germany; e-mail: rollensk@uni-mainz.de

Mathematics Subject Classification (2010): Primary 32G05; Secondary 17B30, 53C30, 32C10 
the Kuranishi space can then be made explicit after the choice of a hermitian metric on $M$. We will go through this construction for our special case of complex parallelisable nilmanifolds in Section 4 .

In general, the Kuranishi space can be arbitrarily singular and non-reduced but we can hope for better control over deformations if we restrict our class of manifolds. If, for example, $X$ is Kähler and has trivial canonical bundle, i.e., $X$ is a Calabi-Yau manifold, then the Tian-Todorov Lemma implies that the Kuranishi space is indeed smooth; we say that $X$ has unobstructed deformations. Such manifolds are very important both in physics and in mathematics, for example in the context of mirror symmetry. The smoothness fails if we drop the Kähler condition [Ghy95].

The only nilmanifolds which can carry a Kähler structure are tori, but nilmanifolds with left-invariant complex structure always have trivial canonical bundle. This follows, as noted in [BDV07], directly from Salamon's work [Sal01, Theorem 3.1] and is also true in a slightly more general context [CG04].

Many known examples like complex tori, the Iwasawa manifold [Nak75], Kodaira surfaces [Bor84], abelian complex structures [CFP06, MPPS06] (see Section 2 for a definition) have indeed unobstructed deformations, and the speculation that a kind of TianTodorov Lemma could be true for large classes of nilmanifolds, including for example hypercomplex structures, was supported by results on weak homological mirror symmetry for nilmanifolds [Poo06, CP08]. On the other hand Catanese and Frediani observed in their study of deformations of principal holomorphic torus bundles, which are in particular nilmanifolds with left-invariant complex structure, that the Kuranishi space can be singular [CF06]. Similar obstructedness phenomena in special examples in real dimension 6 have been noted in [Sal01, Lemma 4.3] and [MPPS06, Section 6].

In this article we want to study deformations in the complex parallelisable case; a lot of other remarkable properties of such manifolds have been described by Winkelmann [Win98]. We will show, in particular, that the Kuranishi space of a complex parallelisable nilmanifold is almost always singular, confirming that even for this well behaved class there is no general or generic unobstructedness result.

Nevertheless, the Kuranishi space cannot become too ugly:

Theorem 4.5. If $X=\Gamma \backslash G$ is a complex parallelisable nilmanifold and $G$ is $v$-step nilpotent, then $\operatorname{Kur}(X)$ is cut out by polynomial equations of degree at most $v$.

In Remark 4.6 we will give an example that the bound on the degree does not remain valid for general nilmanifolds but we believe that there is a larger bound depending on the step length and the dimension only.

It seems that the Lie algebra $\mathfrak{g}$ of $G$ cannot be too far from being free if the Kuranishi space is smooth, and all examples that we found were actually free. Unfortunately, the analysis of the obstructions of higher order becomes complicated but we can at least prove the following:

Theorem 4.8/Corollary 4.9. Let $X=\Gamma \backslash G$ be a complex parallelisable nilmanifold and let $\mathfrak{g}$ be the Lie algebra of $G$. If $\mathfrak{g} /[\mathfrak{g},[\mathfrak{g}, \mathfrak{g}]]$ is not isomorphic to a free 2-step nilpotent Lie algebra then there is a non-vanishing obstruction in degree 2 and the Kuranishi space is singular. 
In particular, if $\mathfrak{g}$ is 2-step nilpotent then $\operatorname{Kur}(X)$ is smooth if and only if $\mathfrak{g}$ is a free 2-step nilpotent Lie algebra.

It is a natural question which infinitesimal deformations in $H^{1}\left(X, \Theta_{X}\right)$ integrate to a 1parameter family of complex parallelisable complex structures. We show in Section 5 that this is the case if and only if they are infinitesimally complex parallelisable; the analogous results holds for abelian complex structures [CFP06].

From this we can also deduce that every complex parallelisable nilmanifold which is not a torus has small deformations which are no longer complex parallelisable (Corollary 5.2). On the other hand it is known that small deformations at least remain in the category of nilmanifolds with left-invariant complex structure (see Section 4 or [ Rol08]).

In Section 6 we will give several examples, mostly in small dimensions. As far as we know, these are the first explicit examples of compact complex manifolds with trivial canonical bundle (or even holomorphic symplectic structure) which have non-reduced Kuranishi space.

\section{Complex parallelisable nilmanifolds and nilmanifolds with left-invariant complex structure}

Let $G$ be a simply connected, complex, nilpotent Lie group with Lie algebra $\mathfrak{g}$, and $\Gamma \subset G$ a lattice, i.e., a discrete cocompact subgroup. By a theorem of Mal'cev [Mal51] such a lattice exists if and only if the real Lie algebra underlying $\mathfrak{g}$ can be defined over $\mathbb{Q}$.

The most important invariant attached to a nilpotent Lie algebra (or Lie group) is its nilpotency index, also called step length, defined as follows: consider the descending central series

$$
\mathcal{C}_{0} \mathfrak{g}:=\mathfrak{g}, \quad \mathcal{C}_{k+1} \mathfrak{g}=\left[\mathcal{C}_{k} \mathfrak{g}, \mathfrak{g}\right] .
$$

Then $\mathfrak{g}$ is nilpotent if and only if there exists a $v$ such that $\mathcal{C}^{v} \mathfrak{g}=0$. The smallest such $v$ is called the nilpotency index.

The quotient $\Gamma \backslash G$ is a complex parallelisable nilmanifold. Such manifolds are equally characterised by the fact that they admit a transitive action of a nilpotent complex Lie group [Wan54].

Nakamura [Nak75] remarked that not all deformations of complex parallelisable nilmanifolds are again complex parallelisable but, as we will discuss in Section 4 , we can describe all deformations in the slightly more general framework of nilmanifolds with left-invariant complex structures.

Let $H$ be a simply connected, real, nilpotent Lie group with Lie algebra $\mathfrak{h}$ and containing a lattice $\Gamma$. Taking the quotient yields a real nilmanifold $M:=\Gamma \backslash H$.

An almost complex structure $J: \mathfrak{h} \rightarrow \mathfrak{h}$ defines an almost complex structure on $H$ by left translation and this almost complex structure is integrable if and only if the Nijenhuis condition

$$
[x, y]-[J x, J y]+J[J x, y]+J[x, J y]=0
$$

holds for all $x, y \in \mathfrak{h}$. In this case we call the pair $(\mathfrak{h}, J)$ a Lie algebra with complex structure. 
The action of $\Gamma$ on the left is then holomorphic and we get an induced complex structure on $M$; we call $(M, J)$ a nilmanifold with left-invariant complex structure.

Note that the multiplication in $H$ induces an action on the left on $M$ if and only if $\Gamma$ is normal if and only if $H=\mathbb{R}^{n}$ is abelian; there is always an action on the right, which is holomorphic if and only if $(H, J)$ is a complex Lie group.

By abuse of notation we will call a vector field, differential form or metric on $M$ left-invariant if its pullback to the universal cover $H$ is left-invariant.

The complexified Lie algebra $\mathfrak{h}_{\mathbb{C}}=\mathfrak{h} \otimes_{\mathbb{R}} \mathbb{C}$ decomposes as

$$
\mathfrak{h}_{\mathbb{C}}=\mathfrak{h}^{1,0} \oplus \mathfrak{h}^{0,1}
$$

where $\mathfrak{h}^{1,0}$ is the $i$-eigenspace of $J$ and $\mathfrak{h}^{0,1}=\overline{\mathfrak{h}^{1,0}}$ is the $(-i)$-eigenspace.

It is not hard to see that the complex structure is integrable if and only if $\mathfrak{h}^{1,0}$ is a (complex) Lie subalgebra of $\mathfrak{h}_{\mathbb{C}}$.

The complex structure $J$ makes $(\mathfrak{h}, J)$ into a complex Lie algebra if and only if the bracket is $J$-linear, i.e., for all $x, y \in \mathfrak{h}$ we have

$$
[J x, y]=J[x, y] .
$$

In this case $H$ is a complex Lie group and $(M, J)$ is complex parallelisable as above. The following equivalent characterisation, which is proved by a straightforward computation using the Nijenhuis tensor (1), is also well known.

Lemma 2.1. A Lie algebra with complex structure $(\mathfrak{h}, J)$ is a complex Lie algebra if and only if $\left[\mathfrak{h}^{1,0}, \mathfrak{h}^{0,1}\right]=0$. In this case the canonical projection

$$
\pi:(\mathfrak{h}, J) \rightarrow \mathfrak{h}^{1,0}, \quad z \mapsto \frac{1}{2}(z-i J z),
$$

is an isomorphism of complex Lie algebras.

Remark 2.2 (Notation). In order to make our notation more transparent, $\mathfrak{h}, H$ and $M$ will always denote a real Lie algebra, Lie group or nilmanifold, often equipped with a (left-invariant) complex structure $J$. We will only consider integrable complex structures.

The notations $\mathfrak{g}, G$ and $X$ will be reserved for their complex parallelisable counterparts. If we need to access the underlying real object with left-invariant complex structure we will write for example $\mathfrak{g}=(\mathfrak{h}, J)$. By the above lemma we can then identify

$$
\mathfrak{g}_{\mathbb{C}}=\mathfrak{h}_{\mathbb{C}}=\mathfrak{g} \oplus \overline{\mathfrak{g}}
$$

where the bracket on $\overline{\mathfrak{g}}$ is given by $[\bar{x}, \bar{y}]=\overline{[x, y]}$ and $[\mathfrak{g}, \overline{\mathfrak{g}}]=\left[\mathfrak{h}^{1,0}, \mathfrak{h}^{0,1}\right]=0$.

Another important class of left-invariant complex structures are so-called abelian complex structures, which are characterised by $\left[\mathfrak{h}^{1,0}, \mathfrak{h}^{1,0}\right]=0$ or, equivalently, $[J x, J y]$ $=[x, y]$ for all $x, y \in \mathfrak{h}$. In some sense this is the opposite condition to being a complex Lie algebra and their deformations have been studied in [MPPS06, CFP06]. As we pointed out in the introduction, deformations behave much more nicely in this case. 


\section{Dolbeault cohomology}

In this section we will describe how the Dolbeault cohomology of a nilmanifold with left-invariant complex structure $(M, J)$ is completely controlled by the Lie algebra with complex structure $(\mathfrak{h}, J)$. This reduces many problems in the study of nilmanifolds to finite-dimensional linear algebra. We will next concentrate on the complex parallelisable case.

Let $(M, J)$ be a nilmanifold with left-invariant complex structure and $\mathfrak{h}$ be the Lie algebra of the corresponding Lie group.

We can identify elements in

$$
\Lambda^{p, q}:=\Lambda^{p, q}\left(\mathfrak{h}^{*}, J\right)_{\mathbb{C}}=\Lambda^{p} \mathfrak{h}^{* 1,0} \otimes \Lambda^{q} \mathfrak{h}^{* 0,1}
$$

with left-invariant differential forms of type $(p, q)$ on $M$. The differential $d=\partial+\bar{\partial}$ restricts to

$$
\Lambda^{*} \mathfrak{h}_{\mathbb{C}}^{*}=\bigoplus \Lambda^{p, q}
$$

and can in fact be defined in terms of the Lie bracket only: for $\alpha \in \mathfrak{h}^{*}$ and $x, y \in \mathfrak{h}$ considered as a differential form and vector fields we have

$$
d \alpha(x, y)=x(\alpha(y))-y(\alpha(x))-\alpha([x, y])=-\alpha([x, y])
$$

since all left-invariant functions are constant.

Let $H^{k}(\mathfrak{h}, \mathbb{C})$ be the $k$-th cohomology group of the complex

$$
\Lambda^{*} \mathfrak{h}_{\mathbb{C}}^{*}: \quad 0 \rightarrow \mathbb{C} \stackrel{0}{\rightarrow} \mathfrak{h}_{\mathbb{C}}^{*} \stackrel{d}{\rightarrow} \Lambda^{2} \mathfrak{h}_{\mathbb{C}}^{*} \stackrel{d}{\rightarrow} \Lambda^{3} \mathfrak{h}_{\mathbb{C}}^{*} \stackrel{d}{\rightarrow} \cdots
$$

and $H^{p, q}(\mathfrak{h}, J)$ be the $q$-th cohomology group of the complex

$$
\Lambda^{p, *}: \quad 0 \rightarrow \Lambda^{p, 0} \stackrel{\bar{\partial}}{\rightarrow} \Lambda^{p, 1} \stackrel{\bar{\partial}}{\rightarrow} \Lambda^{p, 2} \stackrel{\bar{\partial}}{\rightarrow} \cdots
$$

In fact, the first complex calculates the usual Lie algebra cohomology with values in the trivial module $\mathbb{C}$ while the second calculates the cohomology of the Lie algebra $\mathfrak{h}^{0,1}$ with values in the module $\Lambda^{p, 0}$ (see [Rol08]).

Theorem 3.1. Let $M=\Gamma \backslash H$ be a real nilmanifold with Lie algebra $\mathfrak{h}$.

(i) The inclusion of $\Lambda^{*} \mathfrak{h}_{\mathbb{C}}^{*}$ into the de Rham complex induces an isomorphism

$$
H_{\mathrm{dR}}^{*}(M, \mathbb{C}) \cong H^{*}(\mathfrak{h}, \mathbb{C})
$$

in cohomology (Nomizu, [Nom54]).

(ii) The inclusion of $\Lambda^{p, *}$ into the Dolbeault complex induces an inclusion

$$
\iota_{J}: H^{p, q}(\mathfrak{h}, J) \rightarrow H^{p, q}(M, J)
$$

which is an isomorphism if $(M, J)$ is complex parallelisable (Sakane, [Sak76]) or if $J$ is abelian (Console and Fino, [CF01]). Moreover, there exists an open subset $U$ of the space of all left-invariant complex structures on $M$ such that $\iota_{J}$ is an isomorphism for all $J \in U$ ([CF01]). 
Other work in this direction was done by Cordero, Fernández, Gray and Ugarte [CFGU00]. Conjecturally $\iota_{J}$ is an isomorphism for all left-invariant complex structures; in particular no counterexample is known.

For further reference we describe some cohomology groups in these terms. Given $V \subset \mathfrak{g}$ let $\operatorname{Ann}(V):=\left\{\alpha \in \mathfrak{g}^{*}|\alpha|_{V}=0\right\}$ be the annihilator of $V$.

Lemma 3.2. Let $\mathfrak{g}$ be a complex Lie algebra. Denote by $K^{k}:=\operatorname{im}\left(d: \Lambda^{k-1} \mathfrak{h}_{\mathbb{C}}^{*} \rightarrow \Lambda^{k} \mathfrak{h}_{\mathbb{C}}^{*}\right)$ the space of $k$-boundaries. Then

$$
\begin{gathered}
H^{0}(\mathfrak{g}, \mathbb{C})=\mathbb{C}, \quad H^{1}(\mathfrak{g}, \mathbb{C})=\operatorname{Ann}\left(\mathcal{C}_{1} \mathfrak{g}\right)=\operatorname{Ann}([\mathfrak{g}, \mathfrak{g}]), \\
K^{2}=\operatorname{Ann}\left(\operatorname{ker}\left([-,-]: \Lambda^{2} \mathfrak{g} \rightarrow \mathfrak{g}\right)\right) .
\end{gathered}
$$

Moreover, $H^{0,1}(\mathfrak{g})=\overline{H^{1}(\mathfrak{g}, \mathbb{C})}$ and $\operatorname{im}\left(\bar{\partial}: \overline{\mathfrak{g}}^{*} \rightarrow \Lambda^{2} \overline{\mathfrak{g}}^{*}\right)=\bar{K}^{2}$.

Proof. All assertions follow immediately from the fact that the differential $d: \mathfrak{g}^{*} \rightarrow$ $\Lambda^{2} \mathfrak{g}^{*}$ is the dual of the Lie bracket $[-,-]: \Lambda^{2} \mathfrak{g} \rightarrow \mathfrak{g}$ and from the identification $\mathfrak{g}_{\mathbb{C}}=$ $\mathfrak{g} \oplus \overline{\mathfrak{g}}$.

Since we are interested in deformations, the cohomology of the holomorphic tangent bundle (resp. tangent sheaf) $\Theta_{(M, J)}$ is of particular interest. It has been calculated in [Rol08] for left-invariant complex structures for which (4) is an isomorphism, generalising results on abelian complex structure in [MPPS06, CFP06].

But for a complex parallelisable nilmanifold $X$ we can calculate it directly (as observed by Nakamura [Nak75]). Any element of the complex Lie algebra g gives rise to a holomorphic vector field. Hence the tangent sheaf is isomorphic to $\mathcal{O}_{X} \otimes \mathfrak{g}$ and in cohomology we have a natural isomorphism

$$
H^{q}\left(X, \Theta_{X}\right)=H^{q}\left(X, \mathcal{O}_{X} \otimes \mathfrak{g}\right) \cong H^{q}\left(X, \mathcal{O}_{X}\right) \otimes \mathfrak{g}=H^{0, q}(X) \otimes \mathfrak{g} \cong H^{0, q}(\mathfrak{g}) \otimes \mathfrak{g} .
$$

Combining this with the previous results we get

Lemma 3.3. Let $X=\Gamma \backslash G$ be a complex parallelisable nilmanifold. Then the tangent sheaf satisfies $\Theta_{X} \cong \mathcal{O}_{X} \otimes \mathfrak{g}$ and its cohomology is calculated from the complex

$$
0 \rightarrow \mathfrak{g} \stackrel{0}{\rightarrow} \overline{\mathfrak{g}}^{*} \otimes \mathfrak{g} \stackrel{\bar{\partial}}{\rightarrow} \Lambda^{2} \overline{\mathfrak{g}}^{*} \otimes \mathfrak{g} \stackrel{\bar{\partial}}{\rightarrow} \cdots
$$

where the differential of $\bar{\alpha} \otimes X \in \Lambda^{p, 0} \mathfrak{g}$ is given by $\bar{\partial}(\bar{\alpha} \otimes X)=(\bar{\partial} \bar{\alpha}) \otimes X$. In particular

$$
H^{0}(X, \Theta)=\mathfrak{g}, \quad H^{1}(X, \Theta)=H^{1}\left(X, \mathcal{O}_{X}\right) \otimes \mathfrak{g}=\overline{\operatorname{Ann}([\mathfrak{g}, \mathfrak{g}])} \otimes \mathfrak{g} .
$$

\section{Kuranishi theory}

In [Kur62] Kuranishi showed that for every compact complex manifold $X$ there exists a locally complete family of deformations which is versal at $X$. He constructed this family explicitly as a small neighbourhood of zero in the space of harmonic $(0,1)$-forms with values in the holomorphic tangent bundle after choosing some hermitian metric on $X$ (which always exists). 
We will now apply his construction to complex parallelisable nilmanifolds using the results of the last section.

Let $(M, J)=(\Gamma \backslash H, J)$ be the real nilmanifold with left-invariant complex structure underlying a complex parallelisable nilmanifold $X=\Gamma \backslash G$. The complex structure $J$ : $\mathfrak{h} \rightarrow \mathfrak{h}$ is uniquely determined by the eigenspace decomposition $\mathfrak{h}_{\mathbb{C}}=\mathfrak{h}^{1,0} \oplus \mathfrak{h}^{0,1}$.

A (sufficiently small) deformation of this decomposition $\mathfrak{h}_{\mathbb{C}}=V \oplus \bar{V}$ can be encoded in a map $\Phi: \mathfrak{h}^{0,1} \rightarrow \mathfrak{h}^{1,0}$ such that $\bar{V}=(\operatorname{Id}+\Phi) \mathfrak{h}^{0,1}$, i.e., the graph of $\Phi$ in $\mathfrak{h}_{\mathbb{C}}$ is the new space of vectors of type $(0,1)$. This decomposition then determines a unique almost complex structure $J_{V}$, which is integrable if and only if $[V, V] \subset V$.

So far we have only described deformations of $J$ which remain left-invariant; this will be justified in a moment.

The integrability condition is most conveniently expressed using the so-called Schouten bracket: for $X, Y \in \mathfrak{h}^{1,0}$ and $(0,1)$-forms $\bar{\alpha}, \bar{\beta} \in \mathfrak{h}^{* 0,1}$ we set

$$
[\bar{\alpha} \otimes X, \bar{\beta} \otimes Y]:=\bar{\beta} \wedge L_{Y} \bar{\alpha} \otimes X+\bar{\alpha} \wedge L_{X} \bar{\beta} \otimes Y+\bar{\alpha} \wedge \bar{\beta} \otimes[X, Y]
$$

where $L_{X} \bar{\beta}=i_{X} d \bar{\beta}+d\left(i_{X} \bar{\beta}\right)$ is the Lie derivative and $i_{X}$ is the contraction with $X$.

One can then show that the new complex structure is integrable if and only if $\Phi$ satisfies the Maurer-Cartan equation

$$
\bar{\partial} \Phi+[\Phi, \Phi]=0
$$

and it is well known that infinitesimal deformations, which correspond to first-order solutions, are parametrised by classes in $H^{1}\left(X, \Theta_{X}\right)$ (see for example [Cat88] or [Huy05] for an overview). But different solutions may well yield isomorphic deformations.

In order to single out a preferred solution we choose a hermitian structure on $\mathfrak{g}$ which induces a left-invariant hermitian structure on $X$. Using the Hodge star operator associated to the hermitian metric we can define the formal adjoint $\bar{\partial}^{*}$ to $\bar{\partial}$ and the Laplace operator

$$
\Delta:=\bar{\partial}^{*}+\bar{\partial}^{*} \bar{\partial}
$$

Defining the space of harmonic forms to be $\mathcal{H}^{k}=\operatorname{ker}\left(\Delta: \Lambda^{k} \overline{\mathfrak{g}}^{*} \rightarrow \Lambda^{k} \overline{\mathfrak{g}}^{*}\right)$ there is an orthogonal decomposition

$$
\Lambda^{k} \overline{\mathfrak{g}}^{*}=B^{k} \oplus \mathcal{H}^{k} \oplus V^{k}
$$

where $B^{k}=\operatorname{im}\left(\bar{\partial}: \Lambda^{k-1} \overline{\mathfrak{g}}^{*} \rightarrow \Lambda^{k} \overline{\mathfrak{g}}^{*}\right)$ and $V^{k}=\operatorname{im}\left(\bar{\partial}^{*}: \Lambda^{k+1} \overline{\mathfrak{g}}^{*} \rightarrow \Lambda^{k} \overline{\mathfrak{g}}^{*}\right)$; this is just the intersection of the usual Hodge decomposition with the subcomplex of leftinvariant differential forms. The main point is that all harmonic forms are left-invariant in our setting.

Since $\operatorname{ker}(\bar{\partial})=B^{k} \oplus \mathcal{H}^{k}$ we get an isomorphism

$$
H^{k}\left(X, \Theta_{X}\right) \cong H^{k}\left(X, \mathcal{O}_{X}\right) \otimes \mathfrak{g} \cong \mathcal{H}^{k} \otimes \mathfrak{g}
$$


We are especially interested in the first two cohomology groups. By Lemma 3.3 we have $B^{1}=0$, which yields a commutative diagram

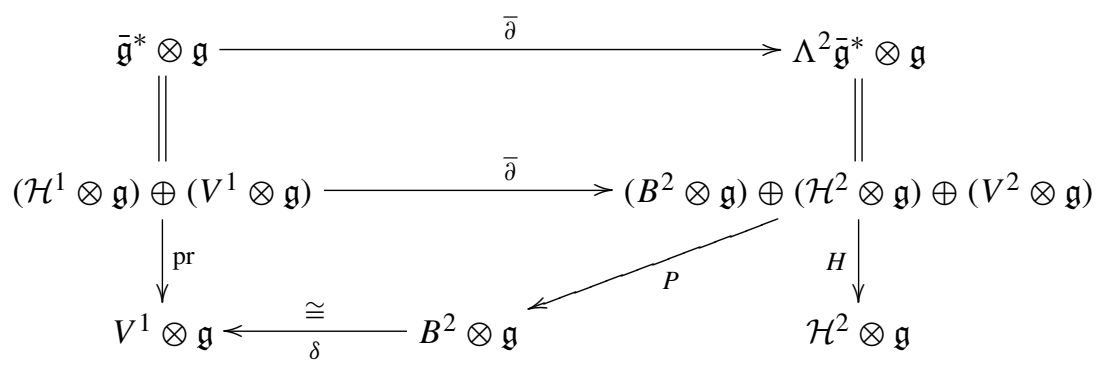

Here $\delta$ denotes the inverse of the isomorphism $P \circ \bar{\partial}: V^{1} \otimes \mathfrak{g} \rightarrow B^{2} \otimes \mathfrak{g}$.

We will now use these operators to describe the Kuranishi space. Let $X_{1}, \ldots, X_{n}$ be a basis of $\mathfrak{g}$ and $\bar{\omega}^{1}, \ldots, \bar{\omega}^{m}$ be a basis for $\mathcal{H}^{1}$. Then $\left\{\bar{\omega}^{i} \otimes X_{j}\right\}$ is a basis of $H^{1}\left(X, \Theta_{X}\right)$ and we define recursively

$$
\begin{aligned}
& \Phi_{1}(\underline{t})=\sum_{i=1}^{m} \sum_{j=1}^{n} t_{i}^{j} \bar{\omega}^{i} \otimes X_{j}, \quad \Phi_{2}(\underline{t}):=-\delta \circ P\left[\Phi_{1}(\underline{t}), \Phi_{1}(\underline{t})\right], \\
& \Phi_{k}(\underline{t}):=-\delta \circ P \sum_{1 \leq i<k}\left[\Phi_{i}(\underline{t}), \Phi_{k-i}(\underline{t})\right] \quad(k \geq 2),
\end{aligned}
$$

obtaining a formal power series $\Phi(\underline{t})=\sum_{k \geq 1} \Phi_{k}(\underline{t})$.

We see that $\Phi_{k}$ is a homogeneous polynomial of degree $k$ in the variables $t_{i}^{j}$ and it is easy to verify that

$$
\bar{\partial} \Phi+[\Phi, \Phi]=H[\Phi, \Phi] .
$$

The map $\Phi$ does not depend on the choice of the basis and we can define the obstruction map

$$
\text { obs : } \mathcal{H}^{1} \otimes \mathfrak{g} \rightarrow \mathcal{H}^{2} \otimes \mathfrak{g}, \quad \mu=\sum_{i, j} t_{i}^{j} \bar{\omega}^{i} \otimes X_{j} \mapsto H[\Phi(\underline{t}), \Phi(\underline{t})] .
$$

We can now formulate Kuranishi's theorem in our context.

Theorem 4.1 ([Kur62]). The formal power series $\Phi(\underline{t})$ converges for sufficiently small values of $t$ and there is a versal family of deformations of $X$ over the space

$$
\operatorname{Kur}(X):=\left\{\mu \in \mathcal{H}^{1}\left(\Theta_{X}\right) \mid\|\mu\|<\epsilon, \operatorname{obs}(\mu)=0\right\}
$$

where $\mathcal{H}^{1}\left(\Theta_{X}\right)=\mathcal{H}^{1} \otimes \mathfrak{g}$ is the space of harmonic 1-forms with values in $\Theta_{X} . \operatorname{Kur}(X)$ is called the Kuranishi space of $X$.

By construction $\Phi$ is left-invariant and hence the new complex structure will also be leftinvariant. In fact, the new subbundle of tangent vectors of type $(0,1)$ in $T M_{\mathbb{C}}$ is obtained by translating the subspace $(\operatorname{Id}+\Phi) \mathfrak{g}^{0,1} \subset \mathfrak{g}_{\mathbb{C}}$. We have reproved that all sufficiently small 
deformations of our complex parallelisable nilmanifold carry a left-invariant complex structure.

Note that the construction involved the choice of a hermitian structure so $\operatorname{Kur}(X)$ is not uniquely defined. Nevertheless for different choices of a metric the germs of the resulting spaces are (non-canonically) isomorphic.

The values of $t$ have to be small for two different reasons. First of all we need to ensure the convergence of the formal power series $\Phi(t)$, and secondly $(\operatorname{Id}+\Phi) \overline{\mathfrak{g}}$ should be the space of $(0,1)$-vectors for an integrable almost complex structure, in other words we need $(\operatorname{Id}+\Phi) \overline{\mathfrak{g}} \oplus \overline{(\operatorname{Id}+\Phi) \overline{\mathfrak{g}}}=\mathfrak{g}_{\mathbb{C}}$. We will see that the first issue does not arise in our setting.

Remark 4.2. Usually the terms of the formal power series $\Phi$ are described using Green's operator, which inverts the Laplacian on the orthogonal complement of harmonic forms:

$$
\Phi_{k}(\underline{t}):=-\bar{\partial}^{*} G \sum_{1 \leq i<k}\left[\Phi_{i}(\underline{t}), \Phi_{k-i}(\underline{t})\right] .
$$

It is straightforward to check that this agrees with our definition above: one uses the identities $G \circ \Delta+H=\Delta \circ G+H=$ Id and the definition of the Laplacian. Our formulation using the map $\delta$ will simplify the computation of examples in Section 6 .

Now that we have seen how the Kuranishi space is constructed we want to investigate its structure in detail for complex parallelisable nilmanifolds.

The key result is the following:

Lemma 4.3. Let $\bar{\alpha} \otimes X, \bar{\beta} \otimes Y \in \overline{\mathfrak{g}}^{*} \otimes \mathfrak{g}$. Then their Schouten bracket is

$$
[\bar{\alpha} \otimes X, \bar{\beta} \otimes Y]=\bar{\alpha} \wedge \bar{\beta} \otimes[X, Y] .
$$

Proof. Comparing the expression with the general formula $[5]$ it suffices to show that for $X \in \mathfrak{g}$ and $\bar{\alpha} \in \overline{\mathfrak{g}}$ the Lie derivative $L_{X} \bar{\alpha}=i_{X} d \bar{\alpha}+d\left(i_{X} \bar{\alpha}\right)$ is zero. But $\bar{\alpha}$ is of type $(0,1)$ and $d \bar{\alpha}$ is of type $(0,2)$ (since $[\mathfrak{g}, \overline{\mathfrak{g}}]=0$ ) so both vanish when contracted with a vector of type $(1,0)$

This gives us

Lemma 4.4. For $\Phi$ as in the recursive description (7) we have

$$
\left[\Phi_{k}, \Phi_{l}\right] \in \Lambda^{2} \overline{\mathfrak{g}}^{*} \otimes \mathcal{C}_{k+l-1} \mathfrak{g} \subset \Lambda^{2} \overline{\mathfrak{g}}^{*} \otimes \mathfrak{g} .
$$

Proof. We argue by induction. By the Jacobi identity, $\left[\mathcal{C}_{k} \mathfrak{g}, \mathcal{C}_{l} \mathfrak{g}\right] \subset \mathcal{C}_{k+l+1} \mathfrak{g}$, and certainly $\Phi_{1} \in \overline{\mathfrak{g}}^{*} \otimes \mathcal{C}_{0} \mathfrak{g}=\overline{\mathfrak{g}}^{*} \otimes \mathfrak{g}$. Since the Schouten bracket is the Lie bracket on the vector part and the map $\delta=\bar{\partial}^{-1}$ acts only on the form part, our claim follows.

We deduce immediately that the Kuranishi space cannot be too complicated:

Theorem 4.5. If $\mathfrak{g}$ is $v$-step nilpotent and $\Phi$ is as in (7) then

$$
\operatorname{obs}(\underline{t})=\sum_{\substack{1 \leq i, j<v, i+j \leq v}} H\left[\Phi_{i}, \Phi_{j}\right] .
$$

In particular $\operatorname{Kur}(X)$ is cut out by polynomial equations of degree at most $v$. 
Proof. Since $\mathfrak{g}$ is $v$-step nilpotent, $\mathcal{C}_{k} \mathfrak{g}=0$ for $k \geq v$. By the previous lemma this implies that $\left[\Phi_{i}, \Phi_{j}\right]=0$ whenever $i+j-1 \geq v$, and hence the only possibly non-vanishing terms of obs $=H[\Phi, \Phi]$ are the ones given above.

Remark 4.6. We believe that also for general nilmanifolds with left-invariant complex structure $(\Gamma \backslash H, J)$ there is some bound (depending only on the Lie algebra $\mathfrak{h}$ ) on the degree of the equations cutting out the Kuranishi space. However it has to be weaker than the one given in Theorem 4.5 as the following example shows.

Consider the complex vector space $V:=\left\langle X_{1}, \ldots, X_{7}\right\rangle_{\mathbb{C}}$. There is a natural real vector space $\mathfrak{h} \subset V \oplus \bar{V}$ with a complex structure such that $\mathfrak{h}^{1,0}=V$.

Let $\omega^{1}, \ldots, \omega^{7} \in V^{*}$ be the dual basis to the $X_{i}$ 's. Then, by the formula for the differential (3), the Lie bracket on $\mathfrak{h}$ is uniquely determined by

$d \omega^{1}=d \omega^{2}=d \omega^{3}=d \omega^{4}=d \omega^{5}=0, \quad d \omega^{6}=\omega^{1} \wedge \omega^{2}, \quad d \omega^{7}=\omega^{3} \wedge \omega^{4}+\bar{\omega}^{1} \wedge \omega^{5}$,

and the complex conjugate equations. The resulting Lie algebra is 2-step nilpotent.

Since $d \mathfrak{h}^{* 1,0} \subset \Lambda^{2,0} \oplus \Lambda^{1,1}$, i.e., $d=\partial+\bar{\partial}$, the complex structure is integrable with respect to this Lie bracket. However, neither is the complex structure abelian, nor is $(\mathfrak{h}, J)$ a complex Lie algebra, because the image of $d$ is not contained in one of the summands $\Lambda^{1,1}$ or $\Lambda^{2,0}$.

The Lie algebra with complex structure $(\mathfrak{h}, J)$ is defined over $\mathbb{Q}$ and by the theorem of Mal'cev [Mal51] there exists a lattice $\Gamma$ in the corresponding real simply connected nilpotent Lie group $H$. We obtain a nilmanifold with left-invariant complex structure $M=(\Gamma \backslash H, J)$.

Using $\bar{\partial} X=[-, X]^{1,0}$ for $X \in \mathfrak{h}^{1,0}$ we see that the element

$$
\Phi_{1}:=\bar{\omega}^{3} \otimes X_{1}+\bar{\omega}^{4} \otimes X_{2}
$$

represents a class in $H^{1}\left(M, \Theta_{M}\right)$; in order to check whether it defines an actual deformation we compute the first terms of the iterative solution (7) of the Maurer-Cartan equation (see Section 6.2.1 for a similar computation).

The Schouten bracket (5) of the infinitesimal deformation is

$$
\left[\Phi_{1}, \Phi_{1}\right]=-\bar{\partial}\left(2 \bar{\omega}^{7} \otimes X_{6}\right)
$$

and hence the second order obstruction vanishes. Setting $\Phi_{2}=2 \bar{\omega}^{7} \otimes X_{6}$ we see that $\left[\Phi_{1}, \Phi_{2}\right]=2 \bar{\omega}^{3} \wedge \bar{\omega}^{5} \otimes X_{6}$ is not $\bar{\partial}$-exact and we have found a non-vanishing obstruction in degree 3 , which is higher than the nilpotency index of $\mathfrak{h}$. Thus the bound in Theorem 4.5 does not hold for general nilmanifolds.

For further reference we use Lemma 4.3 to calculate the second order obstructions, i.e., the quadratic term of the obstruction map obs. Let as before $\bar{\omega}^{1}, \ldots, \bar{\omega}^{m}$ be a basis of $\mathcal{H}^{1}=\overline{\operatorname{Ann}([\mathfrak{g}, \mathfrak{g}])}$ and $X_{1}, \ldots, X_{n}$ be a basis of $\mathfrak{g}$. Then we can represent any element in $H^{1}\left(X, \Theta_{X}\right)$ as

$$
\Phi_{1}(\underline{t})=\sum_{i=1}^{m} \sum_{k=1}^{n} t_{i}^{k} \bar{\omega}^{i} \otimes X_{k}
$$


and consequently

$$
\begin{aligned}
{\left[\Phi_{1}(\underline{t}), \Phi_{1}(\underline{t})\right] } & =\left[\sum_{i, k} t_{i}^{k} \bar{\omega}^{i} \otimes X_{k}, \sum_{j, l} t_{j}^{l} \bar{\omega}^{j} \otimes X_{l}\right] \\
& =\sum_{i, j, k, l}\left(t_{i}^{k} t_{j}^{l}\right)\left[\bar{\omega}^{i} \otimes X_{k}, \bar{\omega}^{j} \otimes X_{l}\right]=\sum_{i, j, k, l}\left(t_{i}^{k} t_{j}^{l}\right) \bar{\omega}^{i} \wedge \bar{\omega}^{j} \otimes\left[X_{k}, X_{l}\right] \\
& =\sum_{1 \leq i<j \leq m} \sum_{k, l}\left(t_{i}^{k} t_{j}^{l}-t_{j}^{k} t_{i}^{l}\right) \bar{\omega}^{i} \wedge \bar{\omega}^{j} \otimes\left[X_{k}, X_{l}\right] \\
& =\sum_{1 \leq i<j \leq m} \sum_{1 \leq k<l \leq n} 2\left(t_{i}^{k} t_{j}^{l}-t_{j}^{k} t_{i}^{l}\right) \bar{\omega}^{i} \wedge \bar{\omega}^{j} \otimes\left[X_{k}, X_{l}\right] \\
& =2 \sum_{1 \leq i<j \leq m} \sum_{1 \leq k<l \leq n} \operatorname{det}\left(\begin{array}{cc}
t_{i}^{k} & t_{i}^{l} \\
t_{j}^{k} & t_{j}^{l}
\end{array}\right) \bar{\omega}^{i} \wedge \bar{\omega}^{j} \otimes\left[X_{k}, X_{l}\right] .
\end{aligned}
$$

We deduce from this formula a necessary condition for the Kuranishi space to be smooth:

Lemma 4.7. If the subspace $\Lambda^{2} \mathcal{H}^{1} \subset \Lambda^{2} \overline{\mathfrak{g}}$ is not contained in $B^{2}$ and $\mathfrak{g}$ is not abelian then there is a non-vanishing obstructions in degree 2 and the Kuranishi space is singular.

Proof. As $\Lambda^{2} \mathcal{H}^{1} \subset \Lambda^{2} \overline{\mathfrak{g}}$ is not contained in $B_{2}$, there is some basis vector $\bar{\omega}^{i} \wedge \bar{\omega}^{j}$ which is not in the image of $\bar{\partial}$. Since $\mathfrak{g}$ is not abelian there are vectors $X_{k}, X_{l}$ such that $\left[X_{k}, X_{l}\right] \neq 0$. Setting $t_{p}^{q}=0$ if $p \neq i, j$ or $q \neq k, l$ and choosing the remaining coefficient such that $\operatorname{det}\left(\begin{array}{cc}t_{i}^{k} & t_{i}^{l} \\ t_{j}^{k} & t_{j}^{l}\end{array}\right) \neq 0$ we have found an obstructed element in $H^{1}\left(X, \Theta_{X}\right)$.

The condition that the Kuranishi space be smooth is very strong. To make this more precise we need to recall the definition of the free 2-step Lie algebra. Let $m \geq 2, V=\mathbb{C}^{m}$ and $\mathfrak{b}_{m}:=V \oplus \Lambda^{2} V$. Then $\mathfrak{b}_{m}$ with the Lie bracket

$$
[\cdot, \cdot]: \mathfrak{b}_{m} \times \mathfrak{b}_{m} \rightarrow \mathfrak{b}_{m}, \quad\left[a+b \wedge c, a^{\prime}+b^{\prime} \wedge c^{\prime}\right]:=a \wedge a^{\prime},
$$

is the free 2-step nilpotent Lie algebra.

Theorem 4.8. If $\mathfrak{g}$ is not abelian then there is a non-vanishing obstruction in degree 2 if and only if $\mathfrak{g} / \mathcal{C}^{2} \mathfrak{g}$ is not isomorphic to the free 2-step nilpotent Lie algebra.

Hence, if $\mathfrak{g} / \mathcal{C}^{2} \mathfrak{g}$ is not free then the Kuranishi space is singular.

The vanishing of all obstructions in degree 2 is not sufficient for the Kuranishi space to be smooth. A 4-dimensional example where $\operatorname{Kur}(X)$ is cut out by a single cubic equation can be found in Section 6.2.1

All examples with smooth Kuranishi space which we could find were actually free Lie algebras and at least in the 2-step nilpotent case there are no other:

Corollary 4.9. If $\mathfrak{g}$ is 2-step nilpotent then the Kuranishi space is smooth if and only if $\mathfrak{g}$ is a free 2-step nilpotent Lie algebra, i.e., $\mathfrak{g} \cong \mathfrak{b}_{m}$ with $m=h^{0,1}(X)$. 
Proof. This follows immediately from the theorem since for a 2 -step nilpotent Lie algebra we have $\mathcal{C}_{2} \mathfrak{g}=0$, hence $\mathfrak{g} / \mathcal{C}_{2} \mathfrak{g} \cong \mathfrak{g}$.

Note that $\mathfrak{b}_{2}$ is the complex Heisenberg algebra, which is the Lie algebra of the universal cover of the Iwasawa manifold. So we have reproved the smoothness of the Kuranishi space of the Iwasawa manifold first observed by Nakamura [Nak75].

It is very easy to produce examples with singular Kuranishi space:

Corollary 4.10. If $\mathfrak{g} \cong \mathfrak{g}^{\prime} \oplus \mathfrak{a}$ where $\mathfrak{a} \cong \mathbb{C}^{n}$ is an abelian Lie algebra and $\mathfrak{g}^{\prime}$ is not abelian, then the Kuranishi space is singular.

In particular, if $X$ is any complex parallelisable nilmanifold which is not a torus and $T$ is a complex torus then $X \times T$ has obstructed deformations.

Proof. We have $\mathfrak{g} / \mathcal{C}_{2} \mathfrak{g}=\mathfrak{g}^{\prime} / \mathcal{C}_{2} \mathfrak{g}^{\prime} \oplus \mathfrak{a}$, which is not free. An application of the theorem proves the assertion.

Before we can address the proof of Theorem 4.8 we need a technical lemma. Let $\mathfrak{g}=$ $\mathcal{C}_{0} \mathfrak{g} \supset \mathcal{C}_{1} \mathfrak{g} \supset \cdots \supset \mathcal{C}_{\nu} \mathfrak{g}=0$ be the descending central series and let $\mathcal{C}^{k} \mathfrak{g}^{*}=\operatorname{Ann} \mathcal{C}_{k} \mathfrak{g}$. We get a filtration

$$
0=\mathcal{C}^{0} \mathfrak{g}^{*} \subset \mathcal{C}^{1} \mathfrak{g}^{*}=\operatorname{Ann}\left(\mathcal{C}_{1} \mathfrak{g}\right) \subset \cdots \subset \mathcal{C}^{\nu} \mathfrak{g}^{*}=\mathfrak{g}^{*}
$$

Lemma 4.11. Considering the vector space

$$
W^{k}:=\left\langle\alpha \wedge \beta \in \Lambda^{2} \mathfrak{g}^{*} \mid \alpha \in \mathcal{C}^{i} \mathfrak{g}^{*}, \beta \in \mathcal{C}^{j} \mathfrak{g}^{*}, i+j \leq k\right\rangle_{\mathbb{C}} \subset \Lambda^{2} \mathfrak{g}^{*}
$$

we have

$$
d \alpha \in W^{k} \Leftrightarrow \alpha \in \mathcal{C}^{k} \mathfrak{g}^{*} .
$$

Proof. Assume that there is $\alpha \notin \mathcal{C}^{k} \mathfrak{g}^{*}$ with $d \alpha \in W^{k}$. By the Jacobi identity, $\mathcal{C}_{k} \mathfrak{g}$ is generated by elements of the form $X=[Y, Z]$ where $Y \notin \mathcal{C}_{1} \mathfrak{g}$ and $Z \in \mathcal{C}_{k-1} \mathfrak{g}$ and hence $\alpha(X) \neq 0$ for one such element. By the definition of $\mathcal{C}^{i} \mathfrak{g}^{*}$ we have $\beta(Y, Z)=0$ for all $\beta \in W^{k}$. On the other hand

$$
d \alpha(Y, Z)=-\alpha([Y, Z])=-\alpha(X) \neq 0
$$

so $d \alpha \notin W_{k}$-a contradiction.

The other direction is a well known fact for nilpotent Lie algebras. It can be easily seen by picking a basis adapted to the descending central series (often called a Mal'cev or Engel basis) and writing $\alpha$ as a linear combination of elements of the dual basis.

Proof of Theorem 4.8. Let $\mathfrak{g}$ be a non-abelian Lie algebra. By Lemma 4.7 it suffices to show that $\Lambda^{2} \mathcal{H}^{1} \subset B^{2} 2$ if and only if $\mathfrak{g} / \mathcal{C}_{2} \mathfrak{g}$ is a free 2-step Lie algebra.

Recalling that $\mathcal{H}^{1}=\overline{\mathcal{C}^{1} \mathfrak{g}^{*}}$ and $B^{2}=\overline{\operatorname{im}(d)}$ we have to prove that $\Lambda^{2} \mathcal{C}^{1} \mathfrak{g}^{*}=W^{1}$ is in the image of the differential if and only if $\mathfrak{g} / \mathcal{C}_{2} \mathfrak{g}$ is free.

The Lie bracket in $\mathfrak{g}$ can also be considered as a linear map

$$
b: \Lambda^{2} \mathfrak{g} \rightarrow \mathcal{C}_{1} \mathfrak{g}
$$


which is, by definition, surjective. Dualising we get (the restriction of) the differential

$$
d:\left(\mathcal{C}_{1} \mathfrak{g}\right)^{*} \rightarrow \Lambda^{2} \mathfrak{g}^{*},
$$

which is now injective.

Let $A$ be the annihilator of $\mathcal{C}_{2} \mathfrak{g}$ in $\left(\mathcal{C}_{1} \mathfrak{g}\right)^{*}$. Then we infer from Lemma 4.11 that $\left.d\right|_{A}$ : $A \rightarrow W^{2}=\Lambda^{2} \mathcal{C}^{1} \mathfrak{g}^{*}$, in fact,

$$
d A=\operatorname{im}(d) \cap W^{2}=\operatorname{im}(d) \cap \Lambda^{2} \mathcal{C}^{1} \mathfrak{g}^{*} .
$$

The dual map

$$
b^{\prime}:\left(\Lambda^{2} \mathcal{C}^{1} \mathfrak{g}^{*}\right)^{*}=\Lambda^{2}\left(\mathfrak{g} / \mathcal{C}_{1} \mathfrak{g}\right) \rightarrow A^{*}=\mathcal{C}_{1} \mathfrak{g} / \mathcal{C}_{2} \mathfrak{g}
$$

gives an anti-symmetric bilinear form on $\mathfrak{g} / \mathcal{C}_{1} \mathfrak{g}$ with values in $\mathcal{C}_{1} \mathfrak{g} / \mathcal{C}_{2} \mathfrak{g}$ which is exactly the Lie bracket in the quotient Lie algebra $\mathfrak{g} / \mathcal{C}_{2} \mathfrak{g}$.

Hence we see that $\Lambda^{2} \mathcal{C}^{1} \mathfrak{g}^{*}$ is in the image of $d$ if and only if $d: A \rightarrow W^{2}$ is surjective if and only if $b^{\prime}$ is injective. But $b^{\prime}$ is by definition surjective so it is injective if and only if it is bijective, in which case $\Lambda^{2}\left(\mathfrak{g} / \mathcal{C}_{1} \mathfrak{g}\right) \cong \mathcal{C}_{1} \mathfrak{g} / \mathcal{C}_{2} \mathfrak{g}$ and the Lie algebra $\mathfrak{g} / \mathcal{C}_{2} \mathfrak{g}$ is indeed free.

\section{Complex parallelisable deformations}

It is a natural question if there are conditions which guarantee that a given small deformation of our complex parallelisable manifold $X$ is again complex parallelisable. So let $\mu \in H^{1}\left(X, \Theta_{X}\right)=\mathcal{H}^{1} \otimes \mathfrak{g}$ be an infinitesimal deformation and $\Phi$ the corresponding iterative solution of the Maurer-Cartan equation as in (7). The new space of $(0,1)$-vectors is $(\mathrm{Id}+\Phi) \overline{\mathfrak{g}}$. (Recall that we identified $\mathfrak{g} \mathbb{C}=\mathfrak{g} \oplus \overline{\mathfrak{g}}$.) if

By Lemma 2.1 the new complex structure is again complex parallelisable if and only

$$
[(\operatorname{Id}+\bar{\Phi}) X,(\operatorname{Id}+\Phi) \bar{Y}]=0
$$

for all $X, Y \in \mathfrak{g}$. Looking at the terms up to first order yields

$$
[X, \bar{Y}]+[\bar{\mu} X, \bar{Y}]+[X, \mu \bar{Y}]=[\bar{\mu} X, \bar{Y}]+[X, \mu \bar{Y}]=0 .
$$

The first of these terms is in $\overline{\mathfrak{g}}$ while the second is in $\mathfrak{g}$, and they are complex conjugate to each other up to sign and renaming. Thus we call $\mu$ an infinitesimally complex parallelisable deformation if

$$
\forall X, Y \in \mathfrak{g}:[X, \mu \bar{Y}]=0 \Leftrightarrow \mu \in \mathcal{H}^{1} \otimes \mathcal{Z} \mathfrak{g},
$$

where $\mathcal{Z} \mathfrak{g}$ denotes the centre of $\mathfrak{g}$. Such infinitesimal deformations are always unobstructed: if $\mu \in \mathcal{H}^{1} \otimes \mathcal{Z} \mathfrak{g}$ then $[\mu, \mu] \in \Lambda^{2} \overline{\mathfrak{g}}^{*} \otimes[\mathcal{Z} \mathfrak{g}, \mathcal{Z} \mathfrak{g}]=0$. Hence in the recursive definition (7) all higher order terms vanish, $\Phi=\mu$ and obs $(\mu)=0$.

We have proved

Theorem 5.1. For an element $\mu \in H^{1}\left(X, \Theta_{X}\right)=H^{1}\left(X, \mathcal{O}_{X}\right) \otimes \mathfrak{g}$ the following are equivalent: 
(i) $\mu \in H^{1}\left(X, \mathcal{O}_{X}\right) \otimes \mathcal{Z} \mathfrak{g}$.

(ii) $\mu$ defines an infinitesimally complex parallelisable deformation.

(iii) $t \mu$ induces a one-parameter family of complex parallelisable manifolds for $t$ small enough, i.e., provided that $(\operatorname{Id}+t \mu) \overline{\mathfrak{g}} \oplus(\operatorname{Id}+t \bar{\mu}) \mathfrak{g}=\mathfrak{g}_{\mathbb{C}}$.

Hence the Kuranishi family is (locally) a cylinder over an analytic subset of the quotient $H^{1}\left(X, \mathcal{O}_{X}\right) \otimes(\mathfrak{g} / \mathcal{Z} \mathfrak{g})$.

For abelian complex structures there is a similar characterisation of infinitesimally abelian deformations [CFP06, Theorem 4].

Since $\mathfrak{g}=\mathcal{Z} \mathfrak{g}$ if and only if $\mathfrak{g}$ is abelian we deduce:

Corollary 5.2. If $\mathfrak{g}$ is not abelian then there are small deformations of $X$ which are not complex parallelisable.

\section{Examples}

We continue to use the notation introduced in Remark 2.2 The deformation theory of the complex parallelisable nilmanifold $X$ is completely determined by the Lie algebra $\mathfrak{g}$ and we have already discussed two series of examples where $\operatorname{Kur}(X)$ is smooth.

- If $\mathfrak{g}=\mathfrak{a}_{k}$ is the $k$-dimensional abelian Lie algebra then $X$ is a torus and $\operatorname{Kur}(X)$ is smooth of dimension $k^{2}(k+1) / 2$.

- If $\mathfrak{g}=\mathfrak{b}_{m}$ is the free 2-step nilpotent Lie algebra on $m$ generators, which has dimension $m(m+3) / 2$, then $\operatorname{Kur}(X)$ is smooth of dimension $m^{2}(m+3) / 2$ (see Corollary 4.9 ).

\subsection{Examples in low dimension-overview}

Nilpotent complex Lie algebras are classified up to dimension 7 [Mag86] and partial results are known in dimension 8. Starting from dimension 7 there are infinitely many non-isomorphic cases.

We will now describe the Kuranishi space of complex parallelisable nilmanifolds up to dimension 5 .

There is a convenient way to describe a nilpotent Lie algebra $\mathfrak{g}$ using the differential $d: \mathfrak{g} \rightarrow \Lambda^{2} \mathfrak{g}$. The expression

$$
\mathfrak{g}=(0,0,0,0,12+34)
$$

means the following: with respect to a basis $\omega^{1}, \ldots, \omega^{5}$ the differential is given by

$$
d \omega^{1}=d \omega^{2}=d \omega^{3}=d \omega^{4}=0 \quad \text { and } \quad d \omega^{5}=\omega^{1} \wedge \omega^{2}+\omega^{3} \wedge \omega^{4} .
$$

This determines the Lie bracket, which is the dual map (see (3)).

More precisely, if we denote by $X_{1}, \ldots, X_{5}$ the dual basis then the only non-zero Lie brackets are $\left[X_{1}, X_{2}\right]=\left[X_{3}, X_{4}\right]=-X_{5}$. 
Table 1. Kuranishi spaces up to dimension 5.

\begin{tabular}{ccccccc}
\hline $\operatorname{dim}$ & Lie algebra & $v$ & $h^{1}\left(\Theta_{X}\right)$ & smooth & irreducible & reduced \\
\hline 1 & $\mathfrak{a}_{1}$ & 1 & 1 & $\sqrt{ }$ & $\sqrt{ }$ & $\sqrt{ }$ \\
\hline 2 & $\mathfrak{a}_{2}$ & 1 & 6 & $\sqrt{ }$ & $\sqrt{ }$ & $\sqrt{ }$ \\
\hline 3 & $\mathfrak{a}_{3}$ & 1 & 18 & $\sqrt{ }$ & $\sqrt{ }$ & $\sqrt{ }$ \\
3 & $\mathfrak{b}_{1}$ & 2 & 6 & $\sqrt{ }$ & $\sqrt{ }$ & $\sqrt{ }$ \\
\hline 4 & $\mathfrak{a}_{4}$ & 1 & 40 & $\sqrt{ }$ & $\sqrt{ }$ & $\sqrt{ }$ \\
4 & $(0,0,0,12)$ & 2 & 12 & - & - & $\sqrt{ }$ \\
4 & $(0,0,12,13)$ & 3 & 8 & - & - & $\sqrt{ }$ \\
\hline 5 & $\mathfrak{a}_{5}$ & 1 & 75 & $\sqrt{ }$ & $\sqrt{ }$ & $\sqrt{ }$ \\
5 & $(0,0,0,12,13)$ & 2 & 15 & - & - & $\sqrt{ }$ \\
5 & $(0,0,0,0,12+34)$ & 2 & 20 & - & - & $\sqrt{ }$ \\
5 & $(0,0,12,13,23)$ & 3 & 10 & $\sqrt{ }$ & $\sqrt{ }$ & - \\
5 & $(0,0,0,12,13+24)$ & 3 & 15 & - & - & - \\
5 & $(0,0,12,13,14)$ & 4 & 10 & - & - & - \\
5 & $(0,0,12,13,14+23)$ & 4 & 10 & - & - & \\
\hline
\end{tabular}

$(v=$ nilpotency index $)$

Table 1 lists all Lie algebras up to dimension 5 in this notation together with some information on the Kuranishi space of an associated complex parallelisable nilmanifold. We denote the nilpotency index by $v$.

Note that all Lie algebras with smooth Kuranishi space are either free or abelian. One can check that also the free 4-step nilpotent Lie algebra on two generators $(0,0,12,13$, $23,14,25,24+15$ ) has smooth Kuranishi space.

\subsection{Examples in low dimension-explicit descriptions}

In this section we will give explicit equations for the Kuranishi space of some examples. In order to avoid cumbersome notation we will only consider the germ of the Kuranishi space at zero which will be denoted by $\operatorname{Kur}(X)_{0}$. Since nothing interesting happens in dimensions 1,2 , and 3 we start in dimension 4.

6.2.1. Computations in dimension 4. We will now compute the Kuranishi space explicitly for the two singular examples in dimension 4.

The structure equations of the Lie algebras in question are given with respect to the bases $X_{1}, \ldots, X_{n}$ and $\omega^{1}, \ldots, \omega^{n}$ as described at the beginning of this section. Thus we will always start the computation of the iterative solution of the Maurer-Cartan equation with the element

$$
\Phi_{1}(\underline{t})=\sum_{i=1}^{m} \sum_{j=1}^{n} t_{i}^{j} \bar{\omega}^{i} \otimes X_{j}
$$

where $n=\operatorname{dim} \mathfrak{g}$ and $m=\operatorname{codim} \mathcal{C}_{1} \mathfrak{g}=h^{0,1}(X)$.

In order to use harmonic forms we equip $\mathfrak{g}$ with the unique hermitian metric such that the $X_{i}$ form an orthonormal basis. 
In every step of the recursion (7) we will decompose $\left[\Phi_{k}, \Phi_{l}\right]=\beta+\chi$ where $\chi$ is harmonic and $\beta$ is exact. Then $\chi$ will contribute to the obstruction map and $\delta(\beta)=$ $(\bar{\partial})^{-1} \beta$ will, if necessary, be used to compute the next iterative step.

The Lie algebra $\mathfrak{g}=(0,0,0,12)$. Since $\mathfrak{g}$ is 2 -step nilpotent we only have to look at obstructions in degree 2 , i.e., obs $=H\left[\Phi_{1}, \Phi_{1}\right]$. Since $\left[X_{1}, X_{2}\right]=-X_{4}$ is the only non-zero bracket we deduce from (8) that

$$
\begin{aligned}
{\left[\Phi_{1}(\underline{t}), \Phi_{1}(\underline{t})\right]=} & -2 \sum_{1 \leq i<j \leq 3} \operatorname{det}\left(\begin{array}{cc}
t_{i}^{1} & t_{i}^{2} \\
t_{j}^{1} & t_{j}^{2}
\end{array}\right) \bar{\omega}^{i} \wedge \bar{\omega}^{j} \otimes X_{4} \\
= & -2 \operatorname{det}\left(\begin{array}{cc}
t_{1}^{1} & t_{1}^{2} \\
t_{3}^{1} & t_{3}^{2}
\end{array}\right) \bar{\omega}^{1} \wedge \bar{\omega}^{3} \otimes X_{4}-2 \operatorname{det}\left(\begin{array}{cc}
t_{2}^{1} & t_{2}^{2} \\
t_{3}^{1} & t_{3}^{2}
\end{array}\right) \bar{\omega}^{2} \wedge \bar{\omega}^{3} \otimes X_{4} \\
& -\bar{\partial}\left(2 \operatorname{det}\left(\begin{array}{cc}
t_{1}^{1} & t_{1}^{2} \\
t_{2}^{1} & t_{2}^{2}
\end{array}\right) \bar{\omega}^{4} \otimes X_{4}\right)
\end{aligned}
$$

Hence

$$
\operatorname{Kur}(X)_{0}=\left\{\underline{t} \in \mathbb{C}^{12} \mid \operatorname{det}\left(\begin{array}{cc}
t_{1}^{1} & t_{1}^{2} \\
t_{3}^{1} & t_{3}^{2}
\end{array}\right)=\operatorname{det}\left(\begin{array}{cc}
t_{2}^{1} & t_{2}^{2} \\
t_{3}^{1} & t_{3}^{2}
\end{array}\right)=0\right\}_{0}=\left(\mathbb{C}^{6} \times Y\right)_{0}
$$

where

$$
Y=\left\{t_{3}^{1}=t_{3}^{2}=0\right\} \cup\left\{\operatorname{rk}\left(\begin{array}{ccc}
t_{1}^{1} & t_{2}^{1} & t_{3}^{1} \\
t_{1}^{2} & t_{2}^{2} & t_{3}^{2}
\end{array}\right) \leq 1\right\} .
$$

In particular we see that the Kuranishi space is a cylinder over the reducible space $Y$.

The Lie algebra $\mathfrak{g}=(0,0,12,13)$ We infer from $(8)$ that

$$
\begin{aligned}
{\left[\Phi_{1}(\underline{t}), \Phi_{1}(\underline{t})\right] } & =-2 \operatorname{det}\left(\begin{array}{cc}
t_{1}^{1} & t_{1}^{2} \\
t_{2}^{1} & t_{2}^{2}
\end{array}\right) \bar{\omega}^{1} \wedge \bar{\omega}^{2} \otimes X_{3}-2 \operatorname{det}\left(\begin{array}{cc}
t_{1}^{1} & t_{1}^{3} \\
t_{2}^{1} & t_{2}^{3}
\end{array}\right) \bar{\omega}^{1} \wedge \bar{\omega}^{2} \otimes X_{4} \\
& =-\bar{\partial}\left(2 \operatorname{det}\left(\begin{array}{cc}
t_{1}^{1} & t_{1}^{2} \\
t_{2}^{1} & t_{2}^{2}
\end{array}\right) \bar{\omega}^{3} \otimes X_{3}+2 \operatorname{det}\left(\begin{array}{cc}
t_{1}^{1} & t_{1}^{3} \\
t_{2}^{1} & t_{2}^{3}
\end{array}\right) \bar{\omega}^{3} \otimes X_{4}\right)
\end{aligned}
$$

and by the recursion formula we set

$$
\Phi_{2}:=2 \operatorname{det}\left(\begin{array}{cc}
t_{1}^{1} & t_{1}^{2} \\
t_{2}^{1} & t_{2}^{2}
\end{array}\right) \bar{\omega}^{3} \otimes X_{3}+2 \operatorname{det}\left(\begin{array}{cc}
t_{1}^{1} & t_{1}^{3} \\
t_{2}^{1} & t_{2}^{3}
\end{array}\right) \bar{\omega}^{3} \otimes X_{4}
$$

We see that there are no obstructions of second order and calculate (noting that $X_{4}$ is in the centre and that $\left[X_{2}, X_{3}\right]=0$ ) 


$$
\begin{aligned}
{\left[\Phi_{1}(\underline{t}), \Phi_{2}(\underline{t})\right] } & =\left[t_{1}^{1} \bar{\omega}^{1} \otimes X_{1}+t_{2}^{1} \bar{\omega}^{2} \otimes X_{1}, 2 \operatorname{det}\left(\begin{array}{cc}
t_{1}^{1} & t_{1}^{2} \\
t_{2}^{1} & t_{2}^{2}
\end{array}\right) \bar{\omega}^{3} \otimes X_{3}\right] \\
& =-2 \operatorname{det}\left(\begin{array}{cc}
t_{1}^{1} & t_{1}^{2} \\
t_{2}^{1} & t_{2}^{2}
\end{array}\right)\left(t_{1}^{1} \bar{\omega}^{1} \wedge \bar{\omega}^{3} \otimes X_{4}+t_{2}^{1} \bar{\omega}^{2} \wedge \bar{\omega}^{3} \otimes X_{4}\right) \\
& =-2 \operatorname{det}\left(\begin{array}{cc}
t_{1}^{1} & t_{1}^{2} \\
t_{2}^{1} & t_{2}^{2}
\end{array}\right)\left(t_{2}^{1} \bar{\omega}^{2} \wedge \bar{\omega}^{3} \otimes X_{4}+t_{1}^{1} \bar{\partial} \bar{\omega}^{4} \otimes X_{4}\right) \\
& =-2 t_{2}^{1} \operatorname{det}\left(\begin{array}{cc}
t_{1}^{1} & t_{1}^{2} \\
t_{2}^{1} & t_{2}^{2}
\end{array}\right) \bar{\omega}^{2} \wedge \bar{\omega}^{3} \otimes X_{4} \bmod B_{2} .
\end{aligned}
$$

Hence we have

$$
\operatorname{Kur}(X)_{0}=\left\{\underline{t} \in \mathbb{C}^{2} \otimes \mathbb{C}^{4}=\mathbb{C}^{8} \mid t_{2}^{1} \operatorname{det}\left(\begin{array}{cc}
t_{1}^{1} & t_{1}^{2} \\
t_{2}^{1} & t_{2}^{2}
\end{array}\right)=0\right\}_{0}
$$

in other words, $\operatorname{Kur}(X)_{0}$ is a cylinder over the cone over the union of a plane and a quadric in $\mathbb{P}^{3}$.

6.2.2. Remarks on dimension 5. The computations in dimension 5 proceed along the same lines as in dimension 4 but are, as one might imagine, much more involved. Thus, we will only present the results.

In view of Theorem 5.1 the Kuranishi space is a cylinder over an analytic subset of the vector space $H^{1}(\overline{\mathfrak{g}}, \mathbb{C}) \otimes(\mathfrak{g} / \mathcal{Z} \mathfrak{g})$ whose dimension we denote by $\mathbf{d}$.

Let $I$ be the ideal generated by the functions cutting out the Kuranishi space. Each ideal in the primary decomposition of $I$ corresponds to an irreducible component of $\operatorname{Kur}(X)_{0}$. Non-reduced components may occur if there are infinitesimal deformations which can be lifted up to a certain order but not to actual deformations. If some component is set-theoretically contained in another component, it is called embedded; such components are always non-reduced.

In all examples the Kuranishi space has several irreducible components. We denote by $\mathbf{k}$ the number of components of the reduced space and by $\mathbf{e}$ the number of embedded components. Note that in the case $\mathfrak{g}=(0,0,12,13,14+23)$ there are two non-reduced components which are not embedded; both are supported on linear subspaces. The results, which were computed using Singular [GPS05], can be found in Table 2, where we also give the codimension and the degree of the various components.

Table 2. Singular deformation spaces in dimension 5 (notation as in Sect.6.2.2).

\begin{tabular}{cccccc}
\hline $\mathfrak{g}$ & $\mathbf{d}$ & $(\mathbf{k}, \mathbf{e})$ & codim & degree & reduced \\
\hline$(0,0,0,12,13)$ & 9 & $(2,0)$ & $(2,2)$ & $(3,1)$ & $(\sqrt{ }, \sqrt{ })$ \\
$(0,0,0,12,13+24)$ & 12 & $(3,2)$ & $(4,5,4) ;(5,5)$ & $(9,3,3) ;(2,4)$ & $(\sqrt{ }, \sqrt{ }, \sqrt{ })$ \\
$(0,0,12,13,14)$ & 8 & $(2,1)$ & $(2,1) ;(2)$ & $(3,1) ;(2)$ & $(\sqrt{ }, \sqrt{ })$ \\
$(0,0,12,13,14+23)$ & 8 & $(4,0)$ & $(2,2,2,2)$ & $(3,2,2,3)$ & $(\sqrt{ }, \sqrt{ },-,-)$ \\
$(0,0,0,0,12+34)$ & 16 & $(2,0)$ & $(5,5)$ & $(20,12)$ & $(\sqrt{ }, \sqrt{ })$ \\
\hline
\end{tabular}


Acknowledgments. The author was supported by a Forschungsstipendium of the DFG. He would like to thank Fabrizio Catanese, Fritz Grunewald, Andrey Todorov and Jörg Winkelmann for several useful comments during a talk at the University of Bayreuth. The referee provided several references which helped to clarify some points in the introduction.

\section{References}

[BDV07] Barberis, M. L., Dotti, I. G., Verbitsky, M.: Canonical bundles of complex nilmanifolds, with applications to hypercomplex geometry. Math. Res. Lett. 16, 331-347 (2009) Zbl 1178.32014 MR 2496748

[Bor84] Borcea, C.: Moduli for Kodaira surfaces. Compos. Math. 52, 373-380 (1984) Zbl 0556.14017 MR 0756728

[Cat88] Catanese, F.: Moduli of algebraic surfaces. In: Theory of Moduli (Montecatini Terme, 1985), Lecture Notes in Math. 1337, Springer, Berlin, 1-83 (1988) Zbl 0658.14017 MR 0963062

[CF06] Catanese, F., Frediani, P.: Deformation in the large of some complex manifolds. II. In: Recent Progress on Some Problems in Several Complex Variables and Partial Differential Equations, Contemp. Math. 400, Amer. Math. Soc., Providence, RI, 21-41 (2006) Zbl pre05049127 MR 2222463

[CG04] Cavalcanti, G. R., Gualtieri, M.: Generalized complex structures on nilmanifolds. J. Symplectic Geom. 2, 393-410 (2004) Zbl 1079.53106 MR 2131642

[CP08] Cleyton, R., Poon, Y. S.: Differential Gerstenhaber algebras associated to nilpotent algebras. Asian J. Math. 12, 225-249 (2008) Zbl pre05363241 MR 2439262

[CF01] Console, S., Fino, A.: Dolbeault cohomology of compact nilmanifolds. Transform. Groups 6, 111-124 (2001) Zbl 1028.58024 MR 1835667

[CFP06] Console, S., Fino, A., Poon, Y. S.: Stability of abelian complex structures. Int. J. Math. 17, 401-416 (2006) Zbl 1096.32009 MR 2220652

[CFGU00] Cordero, L. A., Fernández, M., Gray, A., Ugarte, L.: Compact nilmanifolds with nilpotent complex structures: Dolbeault cohomology. Trans. Amer. Math. Soc. 352, 54055433 (2000) Zbl 0965.32026 MR 1665327

[Ghy95] Ghys, É.: Déformations des structures complexes sur les espaces homogènes de SL(2, C). J. Reine Angew. Math. 468, 113-138 (1995) Zbl 0868.32023 MR 1361788

[GPS05] Greuel, G.-M., Pfister, G., Schönemann, H.: SIngular 3.0, A Computer Algebra System for Polynomial Computations. Centre for Computer Algebra, Univ. of Kaiserslautern (2005); http://www.singular.uni-kl.de

[Huy05] Huybrechts, D.: Complex Geometry. Universitext. Springer, Berlin (2005) Zbl 1055.14001 MR 2093043

[KS58] Kodaira, K., Spencer, D. C.: On deformations of complex analytic structures. I, II. Ann. of Math. (2) 67, 328-466 (1958) Zbl 0128.16901 MR 0112154

[Kur62] Kuranishi, M.: On the locally complete families of complex analytic structures. Ann. of Math. (2) 75, 536-577 (1962) Zbl 0106.15303 MR 0141139

[MPPS06] Maclaughlin, C., Pedersen, H., Poon, Y. S., Salamon, S.: Deformation of 2-step nilmanifolds with abelian complex structures. J. London Math. Soc. (2) 73, 173-193 (2006) Zbl 1089.32007 MR 2197377

[Mag86] Magnin, L.: Sur les algèbres de Lie nilpotentes de dimension $\leq$ 7. J. Geom. Phys. 3, 119-144 (1986) Zbl 0594.17006 MR 0855573

[Mal51] Mal'cev, A. I.: On a class of homogeneous spaces. Amer. Math. Soc. Transl. 39 (1951), 33 pp. MR 0039734 
[Nak75] Nakamura, I.: Complex parallelisable manifolds and their small deformations. J. Differential Geom. 10, 85-112 (1975) Zbl 0297.32019 MR 0393580

[Nom54] Nomizu, K.: On the cohomology of compact homogeneous spaces of nilpotent Lie groups. Ann. of Math. (2) 59, 531-538 (1954) Zbl 0058.02202 MR 0064057

[Poo06] Poon, Y. S.: Extended deformation of Kodaira surfaces. J. Reine Angew. Math. 590, 45-65 (2006) Zbl 1122.32012 MR 2208128

[Rol08] Rollenske, S.: Lie-algebra Dolbeault-cohomology and small deformations of nilmanifolds. J. London Math. Soc. 79, 346-362 (2009) Zbl 1194.32006 MR 2496518

[Sak76] Sakane, Y.: On compact complex parallelisable solvmanifolds. Osaka J. Math. 13, 187212 (1976) Zbl 0361.22005 MR 0422706

[Sal01] Salamon, S. M.: Complex structures on nilpotent Lie algebras. J. Pure Appl. Algebra 157, 311-333 (2001) Zbl 1020.17006 MR 1812058

[Wan54] Wang, H.-C.: Complex parallelisable manifolds. Proc. Amer. Math. Soc. 5, 771-776 (1954) MR 0074064

[Win98] Winkelmann, J.: Complex analytic geometry of complex parallelizable manifolds. Mém. Soc. Math. France (N.S.), 72-73 (1998), 219 pp. Zbl 0918.32015 MR 1654465 